\title{
Penile juvenile xanthogranuloma and neurofibromatosis type-1: risk association with juvenile myelomonocytic leukaemia?
}

K.M. AlGhamdi ${ }^{1}$ and S.N. Al Suwaidan

\section{Introduction}

Juvenile xanthogranuloma (JXG) is a benign, self-healing, non-Langerhans cell histiocytosis characterized by accumulation of lipid-laden macrophages. It occurs most commonly on the head and neck of infants and young children and resolves spontaneously without treatment [1]. Lesions may be single or multiple, congenital or acquired. Size varies from few millimetres to several centimetres.

Two main clinical forms have been described: a small nodular form (60\%) and a large nodular form (40\%). Mixed forms and JXG "en plaque" have been described [2]. Moreover, different morphological types of JXG and atypical locations have been reported $[3,4]$. The first case of penile JXG was reported in 1993 [5].

The typical histologic appearance of JXG consists of a dense dermal infiltrate of foamy histiocytes, foreign body and Touton giant cells, the latter being nearly pathognomonic of JXG [1].

Several authors have reported the occurrence of café-au-lait macules (CALMs) or neurofibromatosis type-1 (NF-1) in patients with JXG $[3,4,6]$. In this report we describe a 7-year-old boy with a solitary penile JXG presentation at age of 2 years in association with NF-1.

\section{Case report}

A 2-year-old boy with multiple CALMs was referred to the dermatology clinic for evaluation of an asymptomatic skin lesion on the penile shaft that has been present for 2 months. The patient was the product of full-term pregnancy and caesarean section delivery due to failure of progression. The neonatal period was uneventful. There was no history of seizures. The mother had an isolated giant CALM on the left lower limb with no other stigmata of neurofibromatosis (NF). The developmental history was appropriate for age. Drug and allergy history were unremarkable.
Physical examination revealed a well-looking baby with stable vital signs and normal growth parameters. No dysmorphic features were noted. Complete cutaneous examination revealed 10 variably sized CALMs ranging in diameter from $3 \mathrm{~mm}$ to $15 \mathrm{~mm}$ with regular borders, and a giant CALM measuring $15 \times 10 \mathrm{~cm}$ on the right thigh (Figure 1). More than 6 of the CALMs measured $\geq 6 \mathrm{~mm}$. No cutaneous tumours, axillary freckling or other stigmata of NF were detected. A well defined, flat-topped, smooth-surfaced, solitary, brown to yellowish, thin plaque measuring $1.5 \times 1.5 \mathrm{~cm}$ was noted on the base of ventral aspect of the penile shaft (Figure 2). Hair, nails and mucous

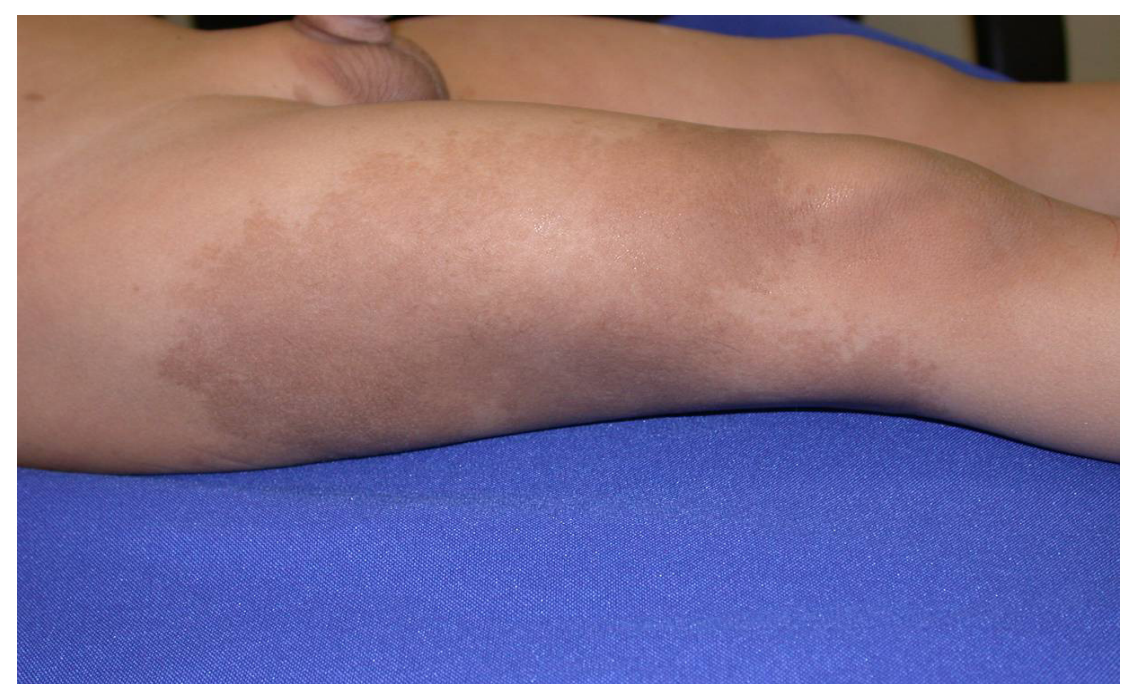

Figure 1 Giant café-au-lait macule involving three-quarters of the lateral aspect of the right thigh $(15 \times 10 \mathrm{~cm})$ 


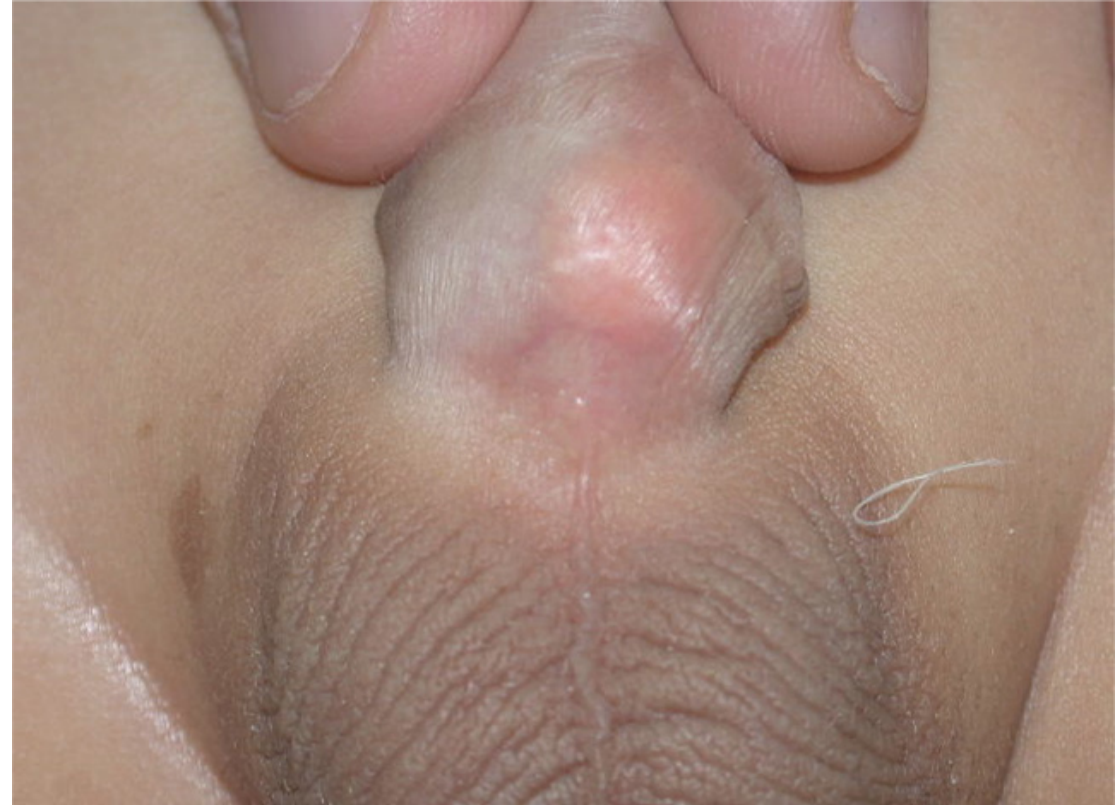

Figure 2 Penile juvenile xanthogranuloma (yellow flat-topped plaque) with adjacent café-au-lait macule

membranes were normal. Ophthalmologic evaluation was within normal limits. The lower left limb was shorter than the right by $2 \mathrm{~cm}$, with resultant limping. The rest of the physical examination was unremarkable.
Skin histopathology of the penile lesion revealed significant dermal infiltrate of foamy histiocytes and eosinophils, however, there were no Touton cells (Figure 3). Laboratory work-up included complete blood count with

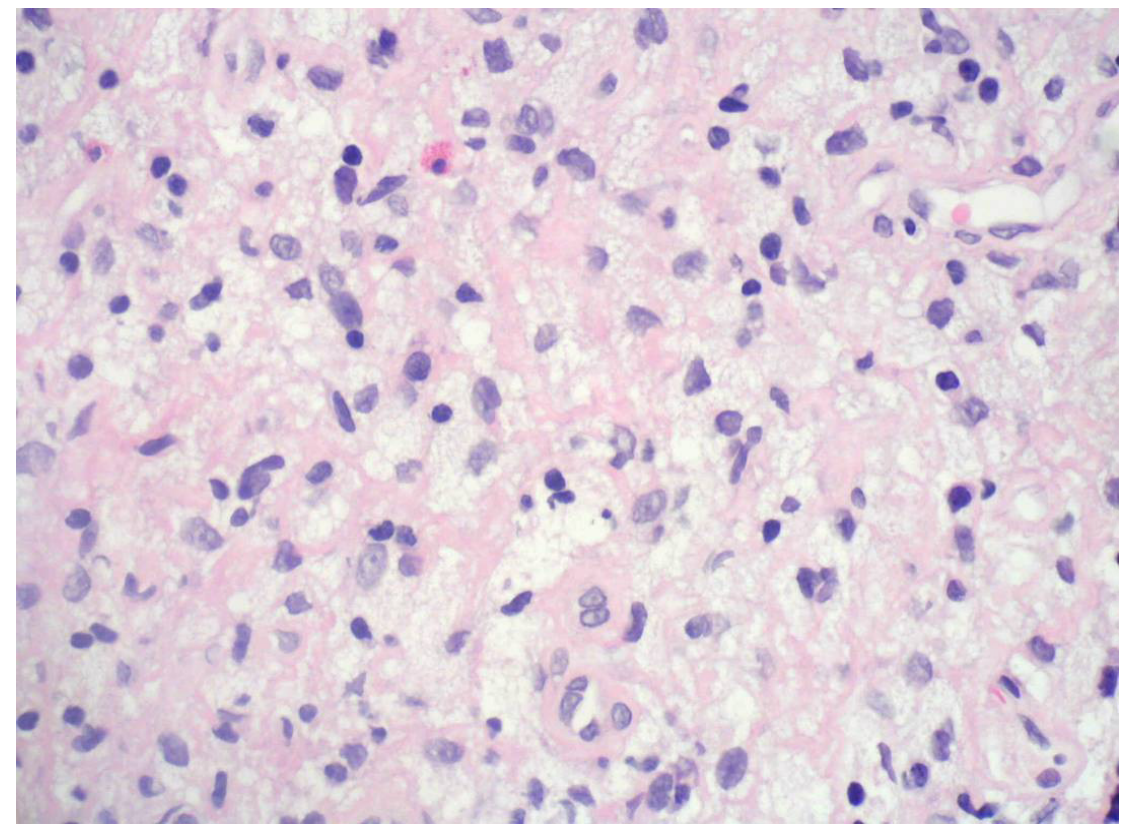

Figure 3 Histopathology of the penile lesion showing significant dermal infiltrate of foamy histiocytes and occasional eosinophils (haematoxylin and eosin) (magnification $\times 40)$ differential, peripheral blood film, renal profile, hepatic profile and random blood sugar: all were within normal limits. X-ray showed that the right femur was $2 \mathrm{~cm}$ longer than the left femur. Spinal X-ray showed widened spinal canal. Brain magnetic resonance imaging showed ill-defined areas of bright signal intensity noted in the pons and medulla oblongata, which is consistent with hamartoma. Based on CALMs, bony changes and CNS hamartoma, this patient was categorized as NF-1. The child is 7 years old now, with 5 years follow-up after the diagnosis of JXG: the JXG lesion resolved completely without residual. So far no symptoms, signs or laboratory changes suggestive of haematological disease have been noted.

We drew the attention of the referring paediatrician and the family to the strong association between JXG and NF-1. The need for regular follow-up with history taking, clinical examination, and haematological work-up was emphasized.

\section{Discussion}

Juvenile xanthogranuloma (JXG) is a benign, self-limiting reactive proliferative disorder of non-Langerhans cell histiocytes seen predominantly in infants and young children and occasionally in adults. Typically it presents with 1 or several papules and nodules located mainly over the face, neck and upper trunk. The lesions are well demarcated, firm and rubbery and vary in diameter from $5 \mathrm{~mm}$ to $20 \mathrm{~mm}$. They are pink to red in the early stages then acquire a yellowish-brown colour later on and may occasionally develop surface telangiectasia. Resolution occurs spontaneously over months to years to leave normal skin, small atrophic scars or hyperpigmentation. Visceral involvement may occur in the eye, lung, liver, spleen, testis, pericardium, gastrointestinal tract, and kidney $[3,4,6,7]$ 
JXG can appear at any body site, including atypical locations such as under a toenail $[8]$, on the penis $[5,9]$, on the clitoral area [10], the eyelids [11], the scrotum $[12,13]$, the lips $[14]$ and the palms and soles [15].

Hautmann and Bachor reported the first case of penile JXG in 1993 in a 2-month-old boy [5]. He had multiple yellow-red nodules on the penile foreskin, penis and glans penis with diameters ranging from $3 \mathrm{~mm}$ to $10 \mathrm{~mm}$. At 1-year follow-up the tumour had disappeared almost completely and the glans penis appeared normal. In our patient, there was solitary penile JXG in the setting of NF-1.

Histopathology of JXG depends on the stage of development. In the early evolutionary stage, the lesion is monomorphous and highly cellular, composed of a sheet like proliferation of small histiocytic cells with some cytoplasmic vacuolation. In the fully developed stage, a more prominent cytoplasmic vacuolization leads to xanthomatized cells and occasional Touton giant cells. Foamy histiocytes become conspicuous as the lesion reaches maturity. If the lesion continues to mature, fibrosis will ensue with an increased number of spindleshaped cells. An inflammatory infiltrate of lymphocytes and eosinophils is common [16]. It has become recognized that JXG can present without foamy histiocytes or giant cells; this is a histologic variant known as non-lipidized JXG $[4,7]$. Nonlipidized JXG represents immature or evolvingJXG lesions. Serial biopsies of a patient with multiple lesions revealed the increasing numbers of foamy histiocytes and giant cells over time [17]. Giant cells tend to be found in the deepest portions of the specimens suggesting that deep biopsies to the subcutaneous layer may facilitate diagnosis $[18,19]$. In our case the Touton giant cells were absent, which may have been related to the immaturity of the lesion or to the superficiality of the biopsy which was done to minimize trauma to this crucial anatomic site, or both.

Nomland reported 3 patients with JXG, 1 of whom had multiple CALMs on the trunk [20]. Whittle reported another patient with JXG and multiple CALMs [21]. Three patients with JXG and CALMs were reported by Marten and Sarkaney [22]. Subsequently, reports describing the association between CALMs and JXG appeared in the literature $[3,4,6]$. To the best of our knowledge, the presented case is the first report of the association between penile JXG and NF-1.

There have been several reports of the association of CALM/NF-1, JXG and juvenile myelomonocytic leukaemia (JMML) formerly known as juvenile chronic myeloid leukaemia (JCML) [23,24]. In 1995 Zvulunov et al. [24] performed a "world statistical analysis" to study this "triple association". They identified cases of JXG with NF-1, of JXG with JMML, of JMML with NF-1, and of all 3 together. They concluded that the triple association was 30-40 times more common than expected and that children with both NF-1 and JXG have a 20-32 times higher risk of developing JMML than do those with NF-1 alone. Since the appearance of their article, the recommendation has frequently been made to screen all children with NF-1 and JXG for JMML. However, a followup letter commenting on the study was critical of the assumptions and statistical calculations [25].

In 2004, a group from Italy reported that out of 288 patients with NF-1, 77 were less than 3 years of age. In this group, 14 children had $\geq 1$ JXGs, but none developed JMML [26]. Despite the fact that it might seem reassuring, such a small number of JXG cases cannot give a solid conclusion. None of the other 63 children under 3 years of age at the time of diagnosis developed JMML and nor did any of the remaining 211 individuals first seen after 3 years of age. SinceJMML is so rare, with an incidence of 1:2000 to 1:5000 in individuals with NF-1, one needs an enormous number of patients to expect to see any JMML. Thus questions about a statistical association between JXG, NF-1, and JMML are unlikely to be answered in a prospective study. Further complicating such a study are the discrepancies in the median age of diagnosis of JXG, JMML, and NF-1, and the need for histologic confirmation of JXG to establish a definite diagnosis [27].

Obviously, the relationship between JXG, NF-1 and JMML is still confusing [27]. Therefore, larger studies are needed to evaluate the true association In the case we have presented there were no symptoms, signs or laboratory changes suggestive of haematological malignancy 5 years after JXG diagnosis.

It is recommended that every physician caring for patients with NF-1 should be aware of the presenting features of JMML. If NF-1 patients develop hepatosplenomegaly, lymphadenopathy or pallor, they should be promptly evaluated for JMML [27]. There is not sufficient information available at this time to recommend approaching children with NF-1 and JXG differently from those who have NF-1 alone. Even if the worst case scenario calculated by Zvulunov et al. [24] is correct, the risk for such a patient would still be much less than $1 \% /$ year, and most of the information generated since 1995 suggests that it is not even that high [27].

In conclusion, a careful, complete ,skin examination must be carried out in any child with JXG, looking particularly for CALMs and other stigmata of NF-1. Paediatricians should be aware of the association between JXG and CALM/ NF-1 and the possible increased risk for JMML. Therefore, in this setting of this association, and until the true risk for JMML is verified, regular follow-up with clinical and laboratory haematological evaluation is recommended. 


\section{References}

1. Roper SS, Spraker MK. Cutaneous histocytosis syndromes. Pediatric dermatology, 1985, 3:19-30.

2. Caputo $\mathrm{R}$ et al. Unusual aspects of juvenile xanthogranuloma. Journal of the American Academy of Dermatology, 1993, 29(5 Pt 2):868-70

3. Herenandez-Martin A et al. Juvenile xanthogranuloma. Journal of the American Academy of Dermatology, 1997, 36(3 Pt 1):355-67.

4. Chand MW. Update of juvenile xanthogranuloma: unusual cutaneous and systemic variants. Seminars in cutaneous medicine and surgery, 1999, 195-205.

5. Hautmann RE and Bachor R. Juvenile xanthogranuloma of the penis. Journal of urology, 1993, 150:456-7.

6. Thami GP, Kaur S, Kanwar AJ. Association of juvenile xanthogranuloma with café-au-lait macules. International journal of dermatology, 2001, 40:283-5.

7. Tahan SR et al. Juvenile xanthogranuloma. Clinical and pathologic characterization. Archives of pathology \& laboratory medicine, 1989, 113:1057-61.

8. Frumkin A, Raytman M, Johson SF. Juvenile xanthogranuloma underneath a toenail. Cutis, 1987, 40:244-5.

9. Freyer DR et al. Juvenile xanthogranuloma: forms of systemic disease and their clinical implications. Journal of pediatrics, 1996, 129(2):227-37.

10. De Villez RL, Limmer BL. Juvenile xanthogranuloma and urticaria pigmentosa. Archives of dermatology, 1975, 111(3):365-6.

11. Schwartz TL et al. Congenital macronodular juvenile xanthogranuloma of the eyelid. Ophthalmology, 1991, 98:1230-3.

12. Jensen NE, Sabharwal S, Walker AE. Nevoxanthoendothelioma and neurofibromatosis. British journal of dermatology, 1971, 86:326-30

13. Morier $\mathbf{P}$ et al. Juvenile chronic granulocytic leukemia, juvenile xanthogranulomas and neurofibromatosis. Journal of the American Academy of Dermatology, 1990, 22:962-5.

14. Torok E, Daroczy J. Juvenile xanthogranuloma an analysis of 45 cases by clinical follow-up, light and electron microscopy. Acto dermato-venereologica, 1985, 65(2):167-9.
15. Kolde G, Bonsmann G. Generalized lichenoid juvenile xanthogranuloma. British journal of dermatology, 1992, 126:66-70.

16. Kubota $Y$ et al. Histopathologic maturation of juvenile xanthogranuloma in a short period. Pediatric dermatology, 2001, 18:127-30.

17. Esterly N, Sahihi T, Medenica M. Juvenile xanthogranuloma. Archives of dermatology, 1997, 105:99-102.

18. Shapiro PE et al. Juvenile xanthogranuloma with inconspicuous or absent foam cells and giant cells. Journal of the American Academy of Dermatology, 1991, 24:1005-9.

19. Newman CC, Raimer SS, Sanchez RL. Non-lipidized juvenile xanthogranuloma: a histologic and immunohistochemical study. Pediatric dermatology, 1997, 14:98-102.

20. Nomland R. Nevoxanthoendothelioma: a benign xanthomatous disease of infants and children Journal of investigative dermatology, 1954, 22:207-15.

21. Whittle CH. Normocholesterolemic xanthomatosis. Transactions of the St. Johns Hospital Dermatological Society, 1958, 41:93.

22. Marten RH, Sarkenay I. Nevoxanthoendothelioma with pigementary abnormalities. British journal of dermatology, 1960, 72:308-11.

23. Morier $\mathrm{P}$ et al. Juvenile chronic granulocytic leukemia ,juvenile xanthogranulomas and neurofibromatosis. Journal of the American Academy of Dermatology, 1990, 22: 962-5.

24. Zvulunov A, Barak Y, Metzker A. Juvenile xanthogranuloma Neurofibromatosis, and juvenile chronic myleiod leukemia. World statistical analysis. Archives of dermatology, 1995, 131:904-8.

25. Gutmann DH, Gurney JG, Shannon KM. Juvenile xanthogranuloma, neurofibromatosis 1, and juvenile chronic myeloid leukemia. Archives of dermatology, 1996, 132:1390.

26. Cambiaghi S, Restano L, Caputo R. Juvenile xanthogranuloma associated with type I neurofibromatosis: 14 patients without evidence of hematologicmalignancies. Pediatric dermatology, 2004, 21(2):97-100.

27. Burgdorf $\mathrm{WH}$, Zelger B. JXG, NF1, and JMML: alphabet soup or a clinical issue? Pediatric dermatology, 2004, 21(2):174-6. 